\title{
FORWARD MODELING OF X-RAY IMAGING CRYSTAL SPECTROMETERS WITHIN THE MINERVA BAYESIAN ANALYSIS FRAMEWORK
}

\begin{abstract}
A. Langenberg, J. Svensson, H. Thomsen, O. Marchuk, N.A. Pablant, R. Burhenn, and R.C. Wolf
Two x-ray imaging crystal spectrometer systems are currently being prepared for commissioning at the stellarator Wendelstein 7-X (W7-X). Both are expected to be ready for the first plasma operation in 2015. The spectrometers will provide line integrated measurements of basic plasma parameters like ion and electron temperatures $\left(T_{e}, T_{i}\right)$, plasma rotation $\left(v_{r o t}\right)$ and argon impurity densities. A forward model based on the designed installation geometries of both spectrometers has been performed using the Minerva Bayesian Analysis framework. This model allows to create synthesized data given radial profiles of plasma parameters for a wide range of different scenarios. For the simulation of line integrated spectra as measured by the (virtual) detector, the geometry and Gaussian detection noise is assumed. The inference of line integrated plasma parameters is done within the framework using the maximum posterior method for an estimation of the plasma parameters from noisy spectral data. Capabilities and limitations of the model and method will be discussed through examples of several synthesized data of different plasma parameter profiles.

Keywords: Bayesian analysis, $x$-ray imaging spectrometer, synthetic diagnostic
\end{abstract}

\section{INTRODUCTION}

X-ray imaging crystal spectrometers have developed to a standard diagnostic for providing line integrated measurements of impurity ion and electron temperatures, poloidal and toroidal plasma rotation, and impurity densities and have been operated successfully at several fusion experiments. ${ }^{1-6}$ In these spectrometers, x-rays emitted from the plasma are imaged via a spherical bent crystal onto a 2D detector unit, monitoring a 2D intensity pattern with energy resolution in horizontal and spatial resolution in vertical direction. A spectral analysis of line integrated data give access to above mentioned plasma parameters.

At W7-X, two imaging spectrometer systems, the High Resolution X-ray Imaging Spectrometer (HR-XIS) and the X-ray Imaging Crystal Spectrometer (XICS) are currently being assembled. The XICS diagnostic has been designed for use with two crystals for a simultaneous measurement of spectral emission of Ar and Fe impurities in different charge states within a wide temperature range $\left(T_{e}=0.3-6 \mathrm{keV}\right)$. For the first operational phase of W7$\mathrm{X}$, the system will be equipped with one crystal with radius of curvature of $1450.4 \mathrm{~mm}$ yielding a dispersion of $2.5 \cdot 10^{-3} \AA / \mathrm{mm}\left(4.3 \cdot 10^{-4} \AA /\right.$ pixel $)$ and a Johann error of $80 \mu \mathrm{m}$ (0.48 pixels). The HR-XIS system can be equipped with up to 8 different crystals, mounted on a rotary stage to measure the emission of several intrinsic or injected impurities, depending on the crystal choice. Thus, in combination with e.g. a pulsed Ar injection, impurity pellet, or a laser blow off system, HR-XIS can be used for dedicated impurity transport studies at W7-X. In this work, a full forward modeling of the HR-XIS and the XICS diagnostic has been performed in the Minerva Bayesian Analysis framework (section II) to validate the expected performance of both spectrometer systems assuming standard as well as advanced plasma scenarios (section III A, B). In addition, a fast spectral fitting rou- tine for inference of line integrated plasma parameters have been carried out. Synthesized data created by a forward modeling of XICS have been used to test the accordance of line integrated $T_{e}$ and $T_{i}$ values with the actual temperature profiles (section III C).

\section{FORWARD MODELING}

A forward modeling of the HR-XIS and XICS diagnostics has been carried out in the Minerva Bayesian Analysis framework. ${ }^{7}$ The framework has proved being a comprehensive and universal tool for modeling a large variety of different diagnostics. ${ }^{8-13}$ It provides several standard routines for spectral emission calculations, line of sight integrations, implementation of VMEC calculations etc. Within this section, a graphical model for modeling measured data of the XICS and HR-XIS diagnostics, assumed temperature and density profiles of several plasma scenarios, and a graphical model for a spectral fitting of measured spectra will be discussed in more detail.

\section{II.A. Graphical Model}

In the Minerva Bayesian Analysis framework, physics parameters and physical models are expressed in a graphical model. Here, model parameters $\boldsymbol{N}$ (blue ellipses), physical models (rectangles), and modeled data $\boldsymbol{D}^{*}$ (gray ellipses, cf. Fig.1) are represented as nodes and linked to each other, defining the dependencies between model parameters and measured data: $f(\boldsymbol{N})=\boldsymbol{D}^{*}$. The use of a graphical model allows for a high flexibility regarding modifications or upgrades of the model. Therefore, even complex rearrangements of the forward model like switching between different spectral emission models, changing line of sight geometries or adding observations from other diagnostics can be handled easily by exchang- 


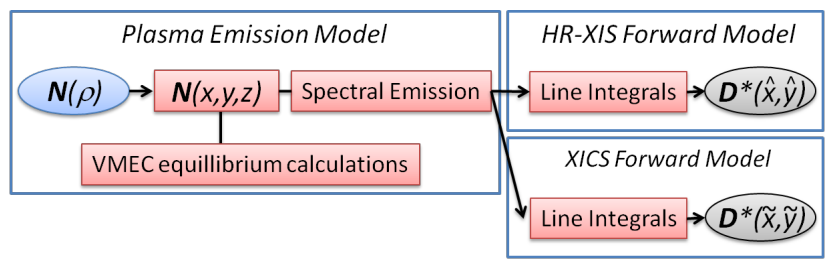

Model Parameters

Physical Models

Modeled Data

FIG. 1. Simplified graphical model of an entire forward model for the HR-XIS and XICS diagnostics.

ing or adding individual nodes. ${ }^{7}$

A simplified scheme of the graphical model used for modeling the x-ray imaging crystal spectrometers is shown in Fig.1. It is split into a physics model (plasma emission model) and two diagnostic models (HR-XIS and XICS forward model). Within the physics model the spectral emission of selected impurities as e.g. He-like Ar can be modeled in 3D space. Input model parameters for evaluation of the spectral emission are radial profiles of ion and electron temperatures $T_{i}$ and $T_{e}$, the electron density $n_{e}$, different ionization stages (H-, He-, and Li-like) of $\operatorname{Ar} n_{A r}^{H / H e / L i}$, and the neutral hydrogen density $n_{H}$, all represented by the blue node in Fig.1. Assuming constance on magnetic flux surfaces, the model parameters are parameterized as a function of the effective radius $\boldsymbol{N}(\rho)$, with $\rho$ being the square root of magnetic flux $\psi$, normalized to the magnetic flux of the last closed flux surface $\psi_{L F}: \rho=\sqrt{\psi / \psi_{L F}}$. For W7-X, the assumption of impurity densities being constant on magnetic flux surfaces is justified since normalized electrostatic potential variations, $e \cdot \Phi / T_{i}$, are below $6 \cdot 10^{-3}$ for relevant collisionalities, ${ }^{14}$ which would result in $<10 \%$ variations in $A r^{16+}$ densities with the electron charge $e$ and the electrostatic potential $\Phi$. If however significant density variations are present, they can be detected by asymmetric impurity density profiles (especially for up down asymmetries) and the model can be adjusted accordingly.

Mapping the 1D profiles $\boldsymbol{N}(\rho)$ into 3D space $\boldsymbol{N}(x, y, z)$ is done using equilibrium flux surfaces $\psi_{n}(x, y, z)$ as given by VMEC calculations, ${ }^{15}$ with spatial coordinates $x, y$, and $z$. Finally, the spectral emission is modeled along lines of sight as defined in the diagnostic model, yielding line integrated synthesized data on the detector (gray nodes in Fig.1) for both HR-XIS and XICS diagnostics. Here, $\hat{x}, \hat{y}$ and $\tilde{x}, \tilde{y}$ denote the detector pixel coordinates. Instrumental effects like the instrumental profile or imaging properties of the crystal are implemented in the diagnostic models via additional nuisance parameters. For instance, the expected dispersion $\delta$ and wavelength calibration $\lambda_{p 0}$ of the spectrometers enter the HR-XIS and XICS forward models (not shown in Fig.1) and can be derived from a spectral fitting, compare Fig.3 and section III C. Spectral lines have been modeled as Voigt profiles with a Gaussian contribution from Doppler broadening and a Lorentz contribution from natural linewidths, convoluted with the instrumental profile. For HR-XIS, the
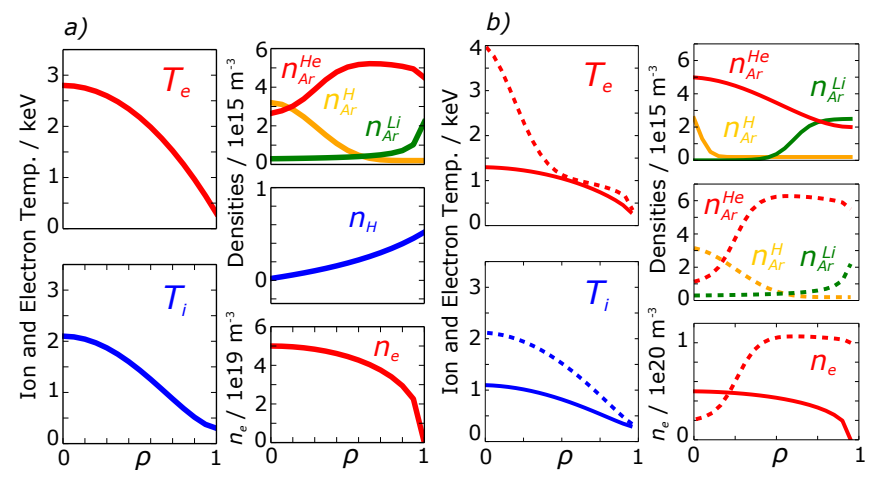

FIG. 2. Assumed temperature and density profiles for calculating the spectral emission of He-like Ar for: a) A standard plasma scenario. b) Low heating power (solid lines) and central density hole scenarios (dotted lines).

instrumental broadening $\left(\lambda_{i}^{F W H M}=5 \cdot 10^{-4} \AA\right)$ compares to the Doppler broadening $\left(\lambda_{D}^{F W H M}=8 \cdot 10^{-4} \AA\right)$ for $T_{i}=0.3 \mathrm{keV}$. Systematic uncertainties of the spatial calibration of lines of sight have been neglected.

Moreover, a flow free plasma has been assumed, neglecting plasma flows parallel as well as perpendicular to the magnetic field lines. While the effect of parallel flows to the measured spectra are expected to be small due to the close to perpendicular orientation of XICS lines of sight to the magnetic axis, perpendicular plasma flows induced by radial electric fields will result in Doppler shifts of spectral emission lines causing an additional line broadening in case of different line shifts at different positions along a line of sight. Given expected perpendicular flow velocities of $v_{p o l} \leq 10 \mathrm{~km} / \mathrm{s}$ at the position of XICS lines of sight, ${ }^{16}$ an upper limit for a potential overestimation of line averaged ion temperatures of $\Delta T_{i} \leq 30 \mathrm{eV}$ can be expected.

\section{II.B. Model Inputs}

For a proper modeling of spectra emitted from highly charged ions, the following atomic effects have been considered in the spectral emission model: A. Excitation of the He-like ground state, B. dielectronic recombination, C. recombination of H-like ions, D. inner-shell excitation and ionization, and E. charge exchange recombination with neutral background gas hydrogen. ${ }^{5}$ The later effect is of particular importance in case of low electron temperatures and a high neutral hydrogen background at the plasma edge as well as for neutral beam injection. ${ }^{17,18}$

In Fig.2, temperature and density profiles of three different plasma scenarios are shown that in the following will be referred to as assumed model parameters utilized for the forward modeling of synthesized data.

The set of temperature and density profiles in Fig.2 a) corresponds to an expected standard plasma scenario of W7-X. Assumed electron and ion temperature and electron density profiles have been set similar to profiles de- 


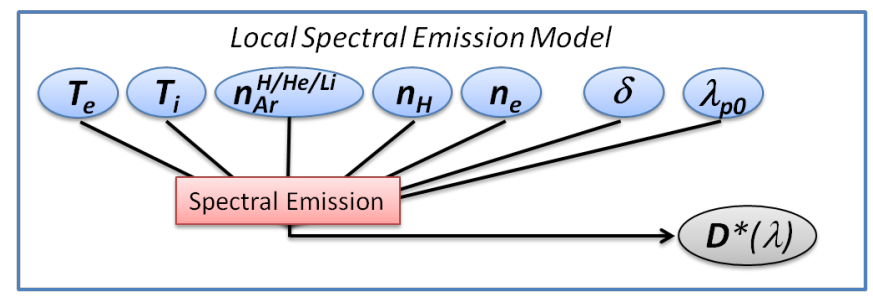

FIG. 3. Graphical model for modeling a single emission spectrum, given the plasma parameters, dispersion $\delta$ of the spectrometer, and absolute wavelength calibration $\lambda_{p 0}$.

rived by neoclassical calculations, ${ }^{19}$ impurity density profiles mimic measured profiles from Alcator C-Mod. ${ }^{3}$

Solid lines in Fig.2 b) represent a set of plasma parameters expected for the first operational phase of W7-X. Since the machine will run in a limiter configuration without a divertor unit, for machine safety reasons short time discharges (several $100 \mathrm{~ms}$ ) with moderate heating power $(\approx 0.5 \mathrm{MW})$ and temperatures $\left(T_{e}<3 \mathrm{keV}\right)$ are going to be realized. Note that due to the low temperatures, the maxima of fractional abundances for the ionization stages of Ar are shifted towards the plasma center. In particular, the hollow $n_{A r}^{H e}$ profile of the standard scenario changes to a peaked profile, see Fig.2 a) and b).

Dotted lines in Fig.2 b) show a set of plasma parameters that are predicted in large stellarators in case of non optimized particle refilling rates. ${ }^{20}$ Here, central electron temperatures are highly peaked up to $4 \mathrm{keV}$ combined with a hollow electron density profile. Additionally, the high central electron temperature will cause the ionization stage of He-like Ar to vanish close to the plasma center, yielding a hollow $n_{A r}^{H e}$ profile as shown in Fig2 b). Such an impurity hole scenario, as observed in LHD, ${ }^{4}$ represents a challenging configuration for $\mathrm{x}$-ray imaging spectrometer systems regarding the inference of plasma parameter profiles, as will be discussed in section III C.

\section{II.C. Spectral Fitting}

In order to infer plasma parameters from measured line integrated spectra, a simplified forward model for modeling a single spectrum given the plasma parameters has been defined in Fig.3. In contrast to the spectrometers forward model, here model parameters $\boldsymbol{N}$ (blue nodes in Fig.3) enter the local emission model as single values and not as radial profiles $\boldsymbol{N}(\rho)$, respectively. Hence, the modeled spectrum (gray node in Fig.3) corresponds to a local spectral emission, given the model parameters $\boldsymbol{N}$. For a spectral fitting of synthesized data, model parameters are varied with respect to a maximization of the posterior $\boldsymbol{P}(\boldsymbol{N} \mid \boldsymbol{D})$, using Bayes theorem:

$$
\boldsymbol{P}(\boldsymbol{N} \mid \boldsymbol{D})=\boldsymbol{P}(\boldsymbol{D} \mid \boldsymbol{N}) \boldsymbol{P}(\boldsymbol{N}) / \boldsymbol{P}(\boldsymbol{D})
$$

with the likelihood distribution $\boldsymbol{P}(\boldsymbol{D} \mid \boldsymbol{N})$, representing the probability of observed data for given parameters $N$, the prior distribution $\boldsymbol{P}(\boldsymbol{N})$ incorporating any knowledge on the model parameters before the measurement took place, and a normalization constant $\boldsymbol{P}(\boldsymbol{D})$. In order to avoid singularities in the forward model, negative values of $\boldsymbol{N}$ have been excluded by setting $\boldsymbol{P}(\boldsymbol{N} \leq \mathbf{0})$ $=0$. For $\boldsymbol{N}>0$, a non informative, flat prior distribution has been assumed, given equal weights for all model parameters.

Applied to spectra observed on a CCD camera with a number of pixels $N_{\text {pix }}$, the likelihood is defined as

$$
\boldsymbol{P}(\boldsymbol{D} \mid \boldsymbol{N})=\prod_{p=0}^{N_{p i x}-1} \frac{1}{\sqrt{2 \pi} \sigma_{p}} \exp \left(-\frac{\left(\boldsymbol{D}_{p}^{*}-\boldsymbol{D}_{p}\right)^{2}}{2 \sigma_{p}^{2}}\right) .
$$

Here, a normal distribution with standard deviation $\sigma_{p}$ is assumed for the photon statistics $\left(\sigma_{p}\right)^{21}$ that is justified for a sufficiently high $(>20)$ number of photon counts. The predicted and observed numbers of counts are $\boldsymbol{D}_{\boldsymbol{p}}^{*}$ and $\boldsymbol{D}_{\boldsymbol{p}}$ for each pixel $\boldsymbol{p}$.

For the calculation of uncertainties of derived plasma parameters $\boldsymbol{N}$, the posterior distribution $\boldsymbol{P}(\boldsymbol{N} \mid \boldsymbol{D})$ has been sampled with a Markov chain Monte Carlo (MCMC) sampling, using a Metropolis Hastings algorithm. ${ }^{2-24}$ Since the accuracy of inferred model parameters are given by the variation of the posterior with respect to the model parameters, sampling of the posterior distribution yields a scatter of model parameters, from which the uncertainty of the model parameters can be calculated.

\section{RESULTS AND DISCUSSION}

\section{III.A. Modeling of the HR-XIS and XICS Diagnostics}

The results of the forward modeling for the HR-XIS and XICS diagnostics are presented in Fig.4. For the forward modeling, assumed temperature and density profiles shown in Fig.2 a) have been used. In Fig.4 a) and c), the designed viewing geometries for both spectrometers are shown in green shaded areas together with the magnetic flux surfaces.

Both spectrometers lines of sight cross the plasma in a poloidal cross section of bean shaped magnetic flux surfaces but at different toroidal angles of $\phi=7.38^{\circ}$ and $\phi=159.09^{\circ}$ for HR-XIS and XICS, respectively. Since the HR-XIS detector consists of six separate CCD detectors, its view field corresponds to 6 arrays of lines of sight as shown in Fig.4 a). The actual cone shaped lines of sight with a maximum vertical expansion of 2 $\mathrm{cm}$ have been approximated by line integrals with a horizontal resolution of $1 \mathrm{~cm}$, equal to 100 points along each line of sight. 
a)

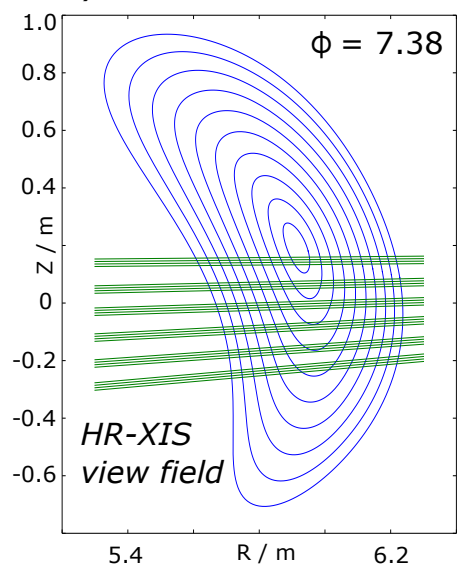

b)

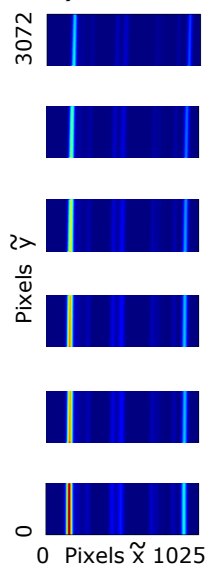

c)

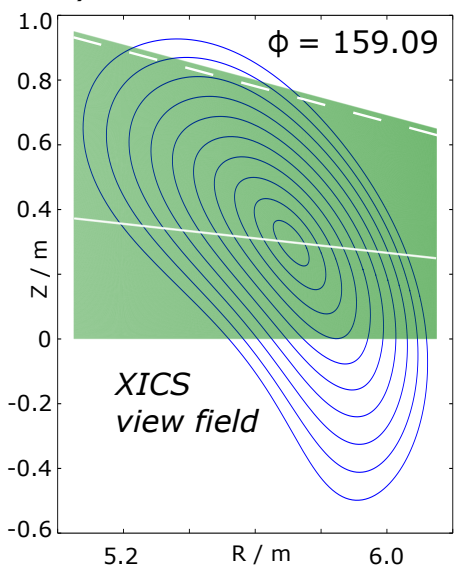

d)

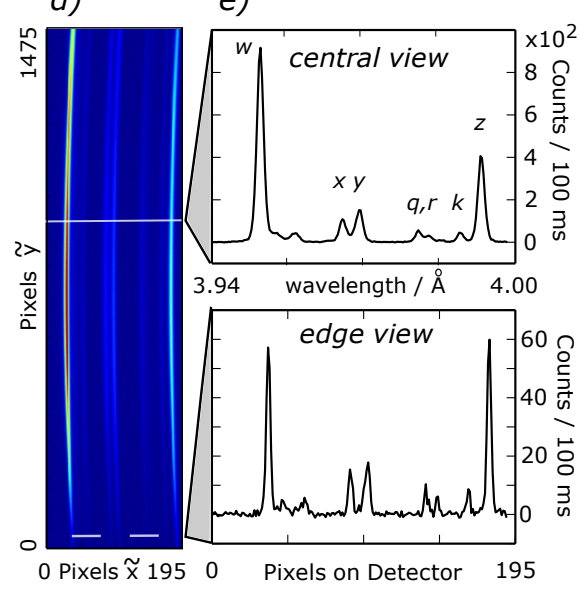

FIG. 4. Forward modeling of the HR-XIS and XICS diagnostics for spectral emission of He-like Ar: a) Magnetic flux surfaces and viewing geometry of HR-XIS in the poloidal cross section of the measurement. b) Modeled 2D intensity pattern on the 6 CCD detector units of HR-XIS. c) Magnetic flux surfaces and viewing geometry of XICS in the poloidal cross section of the measurement. d) Modeled 2D intensity pattern on the CCD detector unit of XICS. Solid and dashed lines correspond to central and edge lines of sight. The uppermost line of sight (edge view) corresponds to the lowest horizontal pixel array (dotted line) and vice versa. e) Synthesized spectra along the central and outermost line of sight with main intense $w, x, y$, and $z$ emission lines and satellites $q, r$, and $k$.

Magnetic flux surfaces in Fig.4 a) and c) shown for $\rho=0.1-1.0$ correspond to VMEC calculations for a vacuum field of a W7-X standard configuration.

Synthesized data at the positions of the HR-XIS and XICS detectors are shown in Fig.4 b) and d). The detector system used for the HR-XIS consists of six CCD3011 deep depletion sensors, Marconi Applied Technologies, England, each with $1024 \times 256$ pixels of $26 \times 26 \mu \mathrm{m}^{2}$ pixel size and an image area of $26.6 \times 6.7 \mathrm{~mm}^{2}$. The XICS diagnostic is equipped with a Pilatus $300 K$, Dectris inc., Switzerland detector with $1475 \times 195$ pixels of $172 \times 172$ $\mu \mathrm{m}^{2}$ pixel size and an image area of $251.4 \times 33.5 \mathrm{~mm}^{2}$. Corresponding to the detector units used in HR-XIS and XICS, a total amount of $1024 \times(6 \times 256)$ and $195 \times 1475$ pixels have been modeled in Fig.4 b) and d).

The 2D intensity patterns on the detectors of the HRXIS and XICS diagnostic have been synthesized for the spectral emission of He-like Ar. In order to simulate a realistic diagnostic environment, noise induced by photon statistics has been added to the expected count rates of both diagnostics. Given the standard plasma scenario shown in Fig. 2 a), count rates in the order of $1 \cdot 10^{5} \mathrm{~s}^{-1}$ are expected for the XICS central line of sight, ${ }^{25}$ taking into account the throughput of the spectrometer, the quantum efficiency of the detector, and the transmission through a Be-foil acting as a vacuum barrier. Expected detector signals shown in Fig.4 and 5 correspond to an integration time of $100 \mathrm{~ms}$.

Fig.4 e) shows line integrated spectra of the XICS diagnostic, averaged along the central and edge line of sight. Within the emission model, the main intense excitation lines $(w, x, y$, and $z)$, dielectronic satellites up to $n=4$, and all inner shell excitation lines (like $q$ and $r$ ) have been taken into account in the spectra as marked in Fig.4 e). Since also the imaging properties of the crystal have been added in the spectral emission model, focal lines on the detector are curved, yielding slight energy shifts between individual lines of sight as can be seen for the central and edge line of sight spectra in Fig.4 e). Possible additional energy shifts induced by plasma rotation have been neglected in this model, see section II A.

\section{III.B. Synthesized XICS Data of Different Plasma Scenarios}

To illustrate the impact of plasma parameters to measured spectra, the forward model has been used to create synthesized spectra of XICS for three different plasma scenarios with assumed plasma parameter profiles shown in Fig.2 a) and b). Fig.5 a)-c) shows synthesized spectra of a) standard, b) low temperature, and c) density hole plasma scenarios for the central and edge lines of sight of XICS.

The individual line intensity ratios as well as the overall intensities of spectral emission lines, especially of the $w$ and $z$ line, are mainly determined by the electron temperature while the linewidths change with the ion temperature. Therefore, radially decreasing electron and ion temperatures towards the edge result in reduced $w / z$ line ratios, reduced overall line intensities, and narrower linewidths for the edge compared to the central line of sight spectra, as can be seen for all plasma scenarios in Fig.5 a) - c).

In spectra of the edge view for the low temperature and density hole scenario, Fig.5 b) and c), even an inverted $w / z$ line ratio $(w / z<1)$ occurs. This effect 

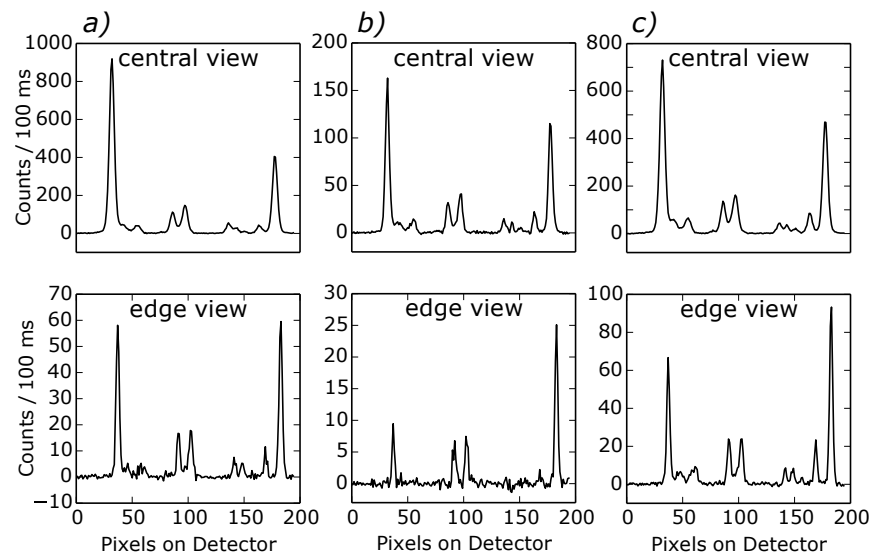

FIG. 5. Synthesized spectra along the central and outermost XICS line of sight for a) standard, b) low temperature, and c) density hole plasma scenarios.

is usually observed at the plasma edge at low electron temperatures ${ }^{3,17,26}$ and is induced by charge exchange of neutral hydrogen background gas with H-like Ar. ${ }^{17}$ For all plasma scenarios the same profile for the neutral hydrogen background has been assumed, see Fig.2 a).

Comparing spectra of the central view for the standard and density hole scenario, Fig.5 a) and c), shows a reduced $w / z$ line ratio for the density hole case, indicating a lower central $T_{e}$ value. However, the $T_{e}$ profile of the density hole scenario is highly peaked and $T_{e}$ in the center is about $1 \mathrm{keV}$ higher than in the standard case. This effect of underestimated central temperatures, induced by the existence of hollow density profiles, will be discussed in more detail in the next section.

\section{III.C. Inference of Line Integrated Ion and Electron Temperatures}

For the inference of plasma parameters out of synthesized XICS data, the fitting routine described in section II $\mathrm{C}$ has been used for line integrated spectra of 20 lines of sight, distributed evenly along the XICS view field. Since thereby just averaged plasma parameters, integrated along the lines of sight can be determined, these line integrated values are not expected to resemble the actual assumed plasma parameter profiles. However, since the assumed profiles are known, the accuracy of line integrated plasma parameters compared to the actual profiles can be evaluated for different plasma scenarios.

Assumed electron and ion temperature profiles (bold solid lines) are shown together with line of sight averaged values (black circles) in Fig.6 for a) standard, b) low temperature, and c) impurity density hole scenarios. Filled and open black circles denote lines of sight above (filled) and below (open) the magnetic axis, gray dots correspond to 500 samples of the posterior distribution for the individual lines of sight. Here, each set of sampled plasma parameters (gray dots, representing the variation of $T_{e}$ and $T_{i}$ values for each line of sight) yields a modeled spectrum that also matches the synthesized one taking into account the photon statistics.

For all plasma scenarios, line integrated temperature profiles are symmetric along the effective radius since inferred temperatures above and below the magnetic axis (filled and open circles) coincide, see Fig.6 a)-c).

In the standard plasma scenario, line of sight averaged $T_{e}$ and $T_{i}$ values are systematically underestimated compared to the assumed profiles, including estimated errors (gray dots). A maximum relative discrepancy compared to assumed profiles of $\delta T_{e}=0.25$ and $\delta T_{i}=0.38$ occurs in the plasma center. Towards the plasma edge, line integrated temperature values gradually approach to the assumed profiles since the region of averaged flux surfaces decrease.

While the uncertainties of line integrated temperatures are dominated by photon statistics, its accuracy is determined by the shapes of $T_{e}$ and $n_{A r}^{H e}$ profiles that determine the spectral emission intensity along the effective radius. If both $T_{e}$ and $n_{A r}^{H e}$ profiles are peaked, the maximum weight of spectral emission is located in the plasma center, yielding a good approximation of line integrated $T_{e}$ and $T_{i}$ values compared to assumed profiles as shown in Fig. $6 \mathrm{~b}$ ). Here, the $n_{A r}^{H e}$ profile has been assumed to be peaked due to the low central electron temperature, see Fig. 2 b). Calculated errors in Fig.6 b) are enhanced for $T_{e}$ and $T_{i}$ compared to Fig. 6 a) due to the lower signal to noise ratio in the low temperature scenario. For the edge line of sight, expected count rates fall below 20 counts and the assumption of a Gaussian likelihood becomes invalid, causing a poor spectral fit of the experimental spectrum that mainly affects the uncertainties of the ion temperature, as can be seen in Fig. 6 b).

If the $n_{A r}^{H e}$ profiles are hollow and close to zero in the plasma center as assumed in the impurity density hole scenario, the central spectral emission intensity will also be close to zero. Therefore, the peaked $T_{e}$ profile is not resembled by the line integrated $T_{e}$ values, shown in Fig.6 c). Calculated errors of $T_{e}$ and $T_{i}$ are slightly reduced compared to the standard plasma scenario of Fig.6 a) due to the higher signal to noise ratio of the density hole scenario, see also Fig.5 a) and c).

Especially for hollow impurity density profiles, the inference of the actual plasma parameter profiles is required. This can be done in principle by using the full spectrometer forward model in Fig.1 for the inference of free parameters, defined as plasma parameter profiles, and will be discussed elsewhere. ${ }^{27}$

As mentioned above, a spectral analysis also yields the wavelength calibration $\lambda_{p 0}$ and the dispersion $\delta$ of the spectrometer. In case of the standard plasma scenario, both parameters can be determined with an uncertainty of $\Delta \lambda_{p 0}=\Delta \delta= \pm 5 \cdot 10^{-5} \AA$, that corresponds to a Doppler shift, created by plasma rotation of $\approx 4 \mathrm{~km} / \mathrm{s}$. In order to infer plasma rotations, an accurate absolute wavelength calibration of the spectrometer is required 


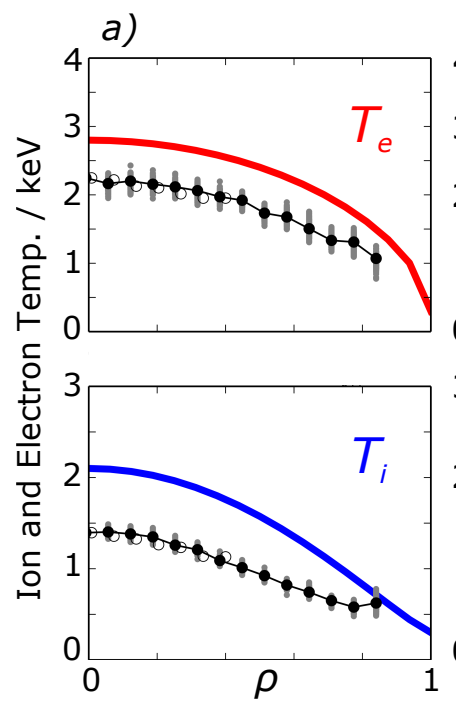

b)

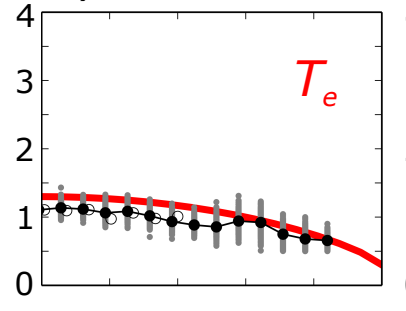

c)
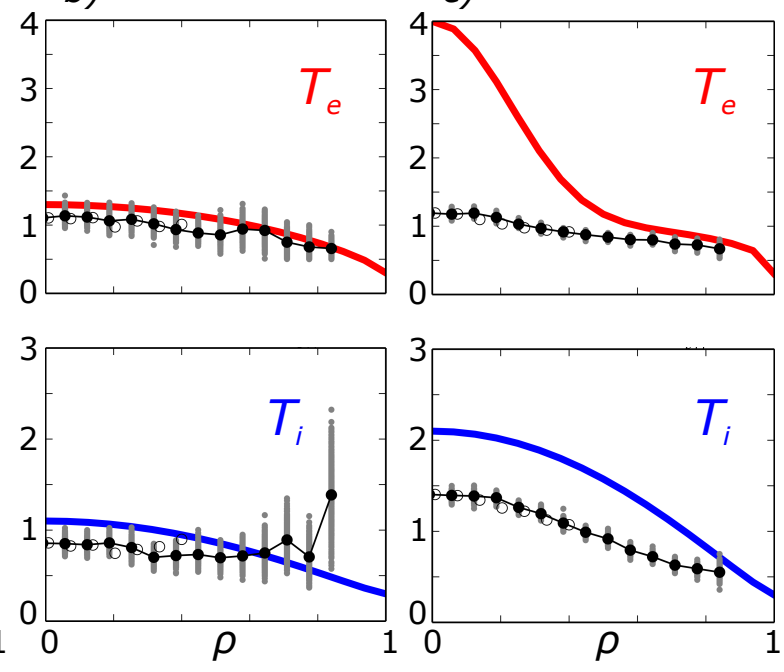

FIG. 6. Assumed electron and ion temperature profiles (solid lines) compared to line integrated values (black circles) inferred from synthesized spectra. Gray dots represent samples from the error distribution indicating the uncertainty of inferred temperatures.

using e.g. plasma discharges with vanishing plasma rotation as reference measurements or an x-ray calibration source.

\section{CONCLUSION}

In this work, a forward modeling of two x-ray imaging spectrometer systems within the Minerva Bayesian Analysis framework has been presented. For different plasma scenarios, synthesized data created by forward modeling of XICS have been fitted with a local spectral emission model to infer line of sight averaged $T_{e}$ and $T_{i}$ values. Since the inference of line of sight averaged values has a fast performance with less than 10 seconds per detector image, it is appropriate for data analysis between plasma shots in the first operation phase of W7-X.

A comparison of line integrated $T_{e}$ and $T_{i}$ values to the actual profiles show a good agreement for peaked $T_{e}$ and $n_{A r}^{H e}$ profiles. In case of hollow impurity density profiles, line integrated temperatures are systematically underestimated and an inversion of line integrated values is required.

\section{ACKNOWLEDGMENTS}

This work has been carried out within the framework of the EUROfusion Consortium and has received funding from the Euratom research and training programme 2014-2018 under grant agreement No 633053. The views and opinions expressed herein do not necessarily reflect those of the European Commission.
${ }^{1}$ A. S. Rosen, M. L. Reinke, J. E. Rice, A. E. Hubbard, and J. W. Hughes, Journal of Physics B-atomic Molecular and Optical Physics 47, 105701 (2014).

${ }^{2}$ S. G. Lee, J. G. Bak, U. W. Nam, M. K. Moon, Y. Shi, M. Bitter, and K. Hill, Review of Scientific Instruments 81, 10 E506 (2010).

${ }^{3}$ A. Ince-Cushman, J. E. Rice, M. Bitter, M. L. Reinke, K. W. Hill, M. F. Gu, E. Eikenberry, C. Broennimann, S. Scott, Y. Podpaly, S. G. Lee, and E. S. Marmar, Review of Scientific Instruments 79, 10E302 (2008).

${ }^{4}$ N. A. Pablant, M. Bitter, L. Delgado-Aparicio, M. Goto, K. W. Hill, S. Lazerson, S. Morita, A. L. Roquemore, D. Gates, D. Monticello, H. Nielson, A. Reiman, M. Reinke, J. E. Rice, and H. Yamada, Review of Scientific Instruments 83, 083506 (2012).

${ }^{5} \mathrm{O}$. Marchuk, Modeling of He-like spectra measured at the tokamaks TEXTOR and TORE SUPRA, Ph.D. thesis, Ruhr Universität Bochum (2004).

${ }^{6}$ P. Beiersdorfer, M. Bitter, M. J. May, and L. Roquemore, Review of Scientific Instruments 74, 1974 (2003).

${ }^{7}$ J. Svensson and A. Werner, in International Symposium on Intelligent Signal Processing-WISP (2007) pp. 955-960.

${ }^{8}$ A. Langenberg, H. Thomsen, R. Burhenn, O. Marchuk, J. Svensson, T. S. Pedersen, and R. C. Wolf, in 41st EPS Conference on Plasma Physics, Vol. 38F (2014) p. 1.074.

${ }^{9}$ S. Schmuck, J. Svensson, L. Figini, T. Jonsson, J. Fessey, L. Meneses, and J. E. Boom, in 41st EPS Conference on Plasma Physics, Vol. 38F (2014) p. 1.025.

${ }^{10}$ D. Li, J. Svensson, H. Thomsen, F. Medina, A. Werner, and R. Wolf, Review of Scientific Instruments 84, 083506 (2013).

${ }^{11}$ O. Ford, Tokamak Plasma Analysis through Bayesian Diagnostic Modelling, Ph.D. thesis, University of London, Imperial College London (2010).

${ }^{12}$ O. Ford, J. Svensson, A. Boboc, and D. C. McDonald, Review of Scientific Instruments 79, 10F324 (2008).

${ }^{13} \mathrm{~J}$. Svensson and A. Werner, Plasma Physics and Controlled Fusion 50, 085002 (2008).

${ }^{14}$ J. M. Garcia-Regana, C. D. Beidler, Y. Turkin, R. Kleiber, P. Helander, H. Maassberg, J. A. Alonso, and J. L. Velasco, "Electrostatic potential variation on the flux surface and its impact on impurity transport." arXiv:1501.03967 (2015).

${ }^{15}$ M. Drevlak, D. Monticello, and A. Reiman, Nuclear Fusion 45, 731 (2005).

${ }^{16}$ J. A. Alonso, Private communication. 
${ }^{17}$ J. E. Rice, E. S. Maramar, E. Källne, and J. Källne, Physical Review A 35, 3033 (1987).

${ }^{18}$ T. Schlummer, O. Marchuk, D. Schultz, G. Bertschinger, W. Biel, D. Reiter, and the TEXTOR-Team, Journal of Physics B: Atomic, Molecular and Optical Physics 48, 144033 (2015).

${ }^{19}$ Y. Turkin, C. D. Beidler, H. Maassberg, S. Murakami, V. Tribaldos, and A. Wakasa, Physics of Plasmas 18, 022505 (2011).

${ }^{20}$ H. Maassberg, C. D. Beidler, and E. E. Simmet, Plasma Physics and Controlled Fusion 41, 1135 (1999).

${ }^{21}$ J. Svensson and R. König, in 32nd EPS Conference on Plasma Physics, Vol. 29C (2005) p. 5.087.
${ }^{22}$ S. Chib and E. Greenberg, American Statistician 49, 327 (1995).

${ }^{23}$ J. Besag, P. Green, D. Higdon, and K. Mengersen, Statistical Science 10, 3 (1995).

${ }^{24}$ R. V. Craiu and J. S. Rosenthal, Annual Review of Statistics and Its Application, Vol 1 1, 179 (2014).

${ }^{25}$ N. A. Pablant, Private communication.

${ }^{26}$ T. Schlummer, O. Marchuk, G. Bertschinger, W. Biel, D. Reiter, and the TEXTOR-Team, in 40th EPS Conference on Plasma Physics, Vol. 37D (2013) p. 5.134.

${ }^{27}$ A. Langenberg, J. Svensson, H. Thomsen, R. Burhenn, R. C. Wolf, O. Marchuk, and N. Pablant, Submitted to Review of Scientific Instruments. 